\title{
Efecto de diferentes láminas de riego y sustratos en la propagación de tomate (Solanum lycopersicum L.)
}

\section{Effect of different water levels and substrates on tomato (Solanum lycopersicum L.) propagation}

YULI DEAQUIZ-OYOLA'

JAVIER ÁLVAREZ-HERRERA', ${ }^{2}$

ANA FRAILE ${ }^{1}$

Frutos del tomate 'Calima'.

Foto: G. Fischer

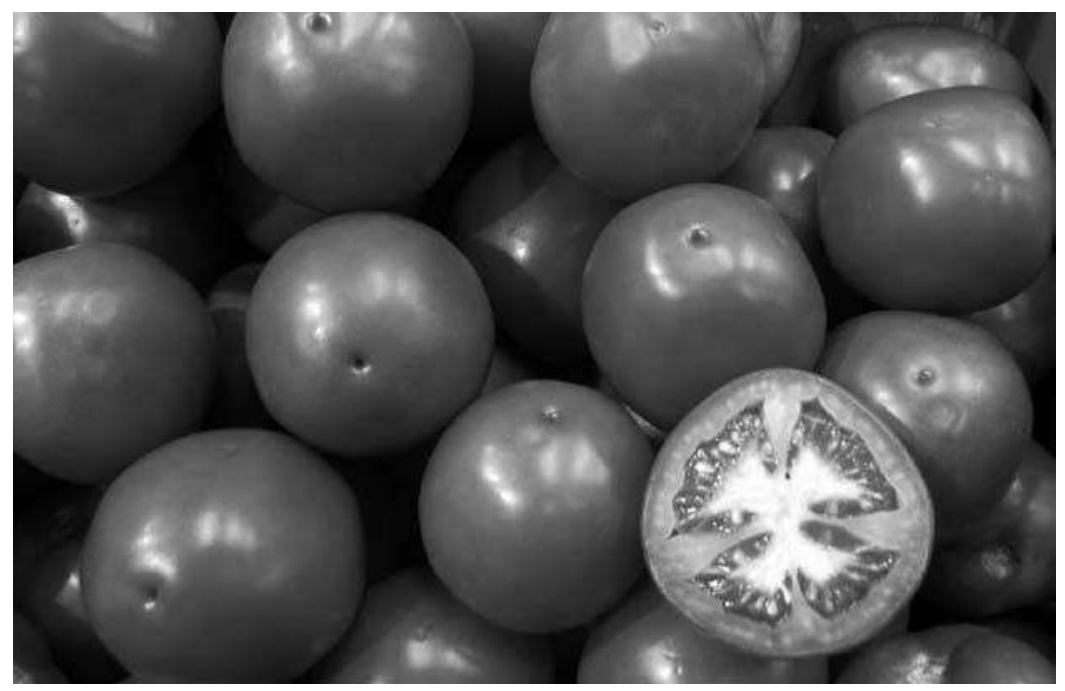

\section{RESUMEN}

Uno de los principales problemas de la producción de tomate es el alto costo de la plantulación, así como el desconocimiento de láminas de riego adecuadas en germinación de semillas. Se evaluó el efecto de diferentes láminas de riego y sustratos en la germinación de semillas del híbrido larga vida Calima, utilizando 25 tratamientos compuestos por las láminas de riego 0,$8 ; 1,0 ; 1,2 ; 1,4$ y 1,6 y los sustratos turba rubia, mezcla de cascarilla de arroz quemado con suelo negro, mezcla de turba rubia con arena, mezcla de turba rubia con cascarilla de arroz quemado $(1: 1 \mathrm{v} / \mathrm{v})$ y mezcla de turba rubia con cascarilla de arroz quemado para un total de 125 unidades experimentales. La combinación de la lámina de riego de 1,2 con la mezcla de turba rubia con cascarilla quemada presentó la mejor respuesta en cuanto a porcentaje, tiempo medio y velocidad media de la germinación, además en altura de la planta, longitud de la raíz, peso en fresco y en seco tanto para las hojas y el tallo. La lámina de riego de 1,6 y el sustrato mezcla de turba rubia con cascarilla quemada superó los demás tratamientos en cuanto área foliar, peso fresco y seco de raíz. El tratamiento óptimo económicamente fue el de la lámina de riego de 1,2 y la mezcla de turba rubia con cascarilla quemada.

1 Grupo de Investigaciones Agrícolas, Facultad de Ciencias Agropecuarias, Universidad Pedagógica y Tecnológica de Colombia (UPTC), Tunja (Colombia).

2 Autor para correspondencia. jgalvarezh@gmail.com 


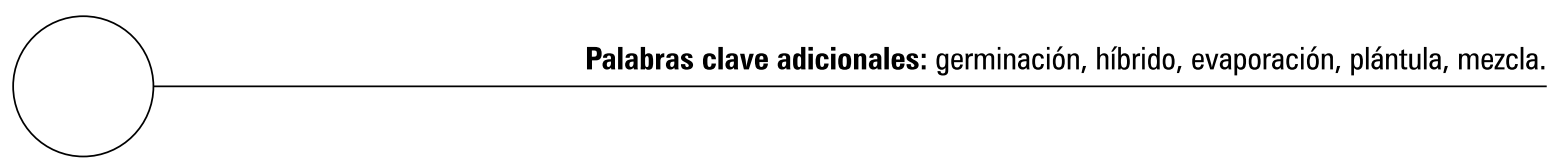

ABSTRACT

Inappropriate substrates and irrigation levels often result in poor seed germination and consequently high priced tomato seedlings. The objective of this study was to assess the effect of different substrates and levels of irrigation on germination of 'Calima' tomato seeds, a long life hybrid. A randomized complete block design was used with 5 replicates and 25 treatments: five irrigation levels $(0.8,1.0,1.2,1.4$ and 1.6 irrigation coefficient) and five substrates (blond peat, a mixture of burned rice husks with black soil, a mixture of blond peat with sand, a mixture of blond peat with rice husks $(1: 1 \mathrm{v} / \mathrm{v})$ and a mixture of blond peat with burned rice husks). The combination of the 1.2 irrigation coefficient and the mixture of peat moss with burned rice husks attained the best response in terms of percentage, time and average rate of germination, seedling height, root length, and fresh and dry weight of leaves and stems. The 1.6 irrigation coefficient and blond peat substrate mixture with rice husks exceeded other treatments in leaf area and fresh and dry weight of the roots. The economically best treatment was that of the 1.2 irrigation coefficient and the substrate mixture of blond peat with burned rice husks

Additional keywords: germination, hybrid, evaporation, seedling, mixture.

Fecha de recepción: 05-02-2008

Aprobado para publicación: 03-06-2008

INTRODUCCIÓN

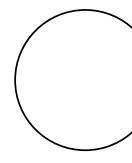

El tomate (Solanum lycopersicum L.) es una de las hortalizas de mayor importancia en el mundo (Gómez et al., 2000). En Colombia es la segunda hortaliza más importante y presenta un rendimiento promedio de $25,78 \mathrm{t} \mathrm{ha}^{-1}$ en el país (FAO, 2007).

La disponibilidad de agua es una condición esencial para la germinación de las semillas. Ésta determina la imbibición y posterior activación de procesos metabólicos, como rehidratación, reparación de membranas y de $\mathrm{ADN}$, elongación celular y aparición de la radícula (Dubreucq et al., 2000). En condiciones naturales, las plantas deben sincronizar sus ciclos de crecimiento y reproducción con un adecuado abastecimiento hídrico (Foley y Fennimore, 1998). La disponibilidad de agua durante el crecimiento de una planta madre afecta el desarrollo de sus semillas, alterando su capacidad germinativa (Pallas et al., 1977; Benech et al., 1992; Gutterman, 2000).

Estudios fisiológicos del proceso de maduración de las semillas muestran que la tolerancia a la desecación es diferente entre las especies y depende principalmente de dos factores: la velocidad a la cual se produce la pérdida de agua y el contenido final después del proceso de desecación (Hong y Ellis, 1992; Ellis y Hong, 1994; Wechsberg et al., 1994; Hay y Probert, 1995).

La aparición de enfermedades y el alto costo de las semillas de tomate mejoradas genéticamente hacen necesaria la utilización de sistemas de producción de plántulas en bandejas de germinación, en las que se utilizan sustratos enriquecidos con fertilizantes (Castilla, 1995). No obstante, el costo de los sustratos importados es alto, por lo 
que surge la necesidad de disponer de materiales producidos localmente que brinden condiciones adecuadas de germinación y de fácil adquisición.

Quesada y Méndez (2005) encontraron que los sustratos que generaron los mejores resultados fueron los que incluían las mezclas de abono orgánico Juan Viñas + granza; lombricompost + abono orgánico Juan Viñas + granza y abono orgánico Juan Viñas, en almácigos de brócoli, pepino y tomate.

Los experimentos desarrollados para evaluar el efecto de diferentes tipos de vermicompost sobre la germinación, crecimiento, floración y fructificación de varias especies hortícolas y ornamentales como pepino (Cucumis sativus L.), tomate (Solanum lycopersicum L.), petunias (Petunia grandiflora L.), maravillas (Calendula officinalis L.), crisantemos (Chysanthemum sinense L.) demostraron que las mejores respuestas de estas especies se presentan cuando se sustituye del $10 \%$ al $20 \%$ del volumen total del medio de crecimiento comercial con los diferentes tipos de vermicompost (Riggle, 1998; Subler et al., 1998).

Por lo anterior, el objetivo de este trabajo fue evaluar el efecto de diferentes láminas y sustratos en germinación y obtención de plántulas de un híbrido de tomate larga vida, para asegurar una óptima calidad y eficiencia, reduciendo costos y optimizando el proceso de propagación.

\section{MATERIALES Y MÉTODOS}

El experimento se realizó en el invernadero de vidrio de la Facultad de Ciencias Agropecuarias de la Universidad Pedagógica y Tecnológica de Colombia en la ciudad de Tunja, $5^{\circ} 32^{\prime} \mathrm{N}, 73^{\circ}$ $23^{\prime}$ W y 2.782 msnm. La temperatura promedio dentro del invernadero fue de $20^{\circ} \mathrm{C}$ y la humedad relativa del $65 \%$.
Como material vegetal, se empleó el híbrido de tomate (Solanum lycopersium L.) larga vida 'Calima', el cual se seleccionó y se sembró en bandejas de polietileno de 72 alvéolos. La lámina de riego suministrada correspondió a cinco coeficientes de la evaporación (Ev), los sustratos empleados para la germinación fueron turba rubia canadiense (TR), mezcla de cascarilla de arroz quemado con suelo negro (COSN), mezcla de turba rubia con arena (TRA), mezcla de turba rubia con cascarilla de arroz (TRCA) y mezcla de turba rubia con cascarilla de arroz quemado (TRCQ), todas las mezclas se hicieron en proporción 1:1 en volumen.

El diseño estadístico utilizado fue completamente al azar. Se evaluó la combinación de cinco diferentes sustratos y cinco láminas de riego $(0,8$; $1,0 ; 1,2 ; 1,4$ y 1,6$)$ para un total de 25 tratamientos (tabla 1), con cinco repeticiones, para un total 125 unidades experimentales (UE).

La aplicación del primer riego se inició luego de la emergencia de las plántulas. Se instaló dentro del invernadero un evaporímetro plástico a escala del tanque tipo A con dimensiones de 29 $\mathrm{cm}$ de diámetro y $6,1 \mathrm{~cm}$ de profundidad, realizando lecturas diarias de la evapotranspiración (Etp). Para el suministro de agua a la planta, se utilizó una jeringa de $10 \mathrm{~mL}$ con el fin de tener una mayor exactitud en las cantidades aplicadas, utilizando la siguiente fórmula:

$$
\text { Lamina }=\frac{\text { Etp }^{*} \mathrm{C}^{*} \mathrm{~A}}{\eta_{\mathrm{r}}}
$$

En la cual, Etp = evapotranspiración en mm medida en el tanque evaporímetro; $\mathrm{C}=$ coeficiente multiplicador; $\mathrm{A}=$ área del alvéolo; $\eta_{\mathrm{r}}=$ eficiencia del riego $(0,9)$.

La aplicación del primer riego se realizó luego de la emergencia de las plántulas. En la etapa de 
Tabla 1. Tratamientos del estudio.

\begin{tabular}{|c|l|c|}
\hline Tratamientos & Sustratos & Coeficiente de riego \\
\hline T1 & COSN & 1,6 \\
\hline T2 & COSN & 1,0 \\
\hline T3 & COSN & 0,8 \\
\hline T4 & COSN & 1,2 \\
\hline T5 & COSN & 1,4 \\
\hline T6 & TRA & 1,6 \\
\hline T7 & TRA & 1,0 \\
\hline T8 & TRA & 0,8 \\
\hline T9 & TRA & 1,2 \\
\hline T10 & TRA & 1,4 \\
\hline T11 & TR & 1,6 \\
\hline T12 & TR & 1,0 \\
\hline T13 & TR & 0,8 \\
\hline T14 & TR & 1,2 \\
\hline T15 & TR & 1,4 \\
\hline T16 & TRCA & 1,6 \\
\hline T17 & TRCA & 1,0 \\
\hline T18 & TRCA & 0,8 \\
\hline T19 & TRCA & 1,2 \\
\hline T20 & TRCA & 1,4 \\
\hline T21 & TRCO & 1,6 \\
\hline T22 & TRCO & 1,0 \\
\hline T23 & TRC0 & 0,8 \\
\hline T24 & TRC0 & 1,2 \\
\hline T25 & TRC0 & \\
\hline & & 1,4 \\
\hline
\end{tabular}

Abreviaturas

COSN: mezcla de cascarilla de arroz quemado con suelo negro.

TRA: mezcla de turba rubia con arena.

TR: Turba rubia.

TRCA: mezcla de turba rubia con cascarilla de arroz.

TRCQ: mezcla de turba rubia con cascarilla de arroz quemado.

plántula, se midió área foliar, mediante el analizador Li-Cor® 3000A (Lincoln, NE); peso fresco y seco $\left(70^{\circ} \mathrm{C}\right.$ durante $\left.48 \mathrm{~h}\right)$, de raíz, tallo y hojas, longitud de raíz principal, altura final, eficiencia de tratamientos que se calcularon con base en la relación peso seco de plántula/consumo de agua y análisis económico que se calculó con base en el costo de tratamiento.
Se realizaron los análisis de varianza (Anova) con el fin de determinar la existencia de diferencias estadísticas entre sustratos y láminas de riego, asimismo mediante la prueba de comparación de Tukey con una confiabilidad del $95 \%$, se establecieron los mejores tratamientos. Este análisis se realizó en el programa SAS v. 8.1e (Cary, NC).

\section{RESULTADOS Y DISCUSIÓN}

\section{Germinación}

El porcentaje de germinación presentó diferencias significativas entre tratamientos, la mejor respuesta se encontró con el sustrato TRCQ y un coeficiente de riego de 1,2 (figura 1). Entre sustratos, se observaron diferencias significativas, pero no entre láminas de riego; la mezcla TRCQ dio el mejor resultado. Debido a que el sustrato tuvo mayor retención de humedad y mayor espacio poroso, lo que permite una adecuada disponibilidad de oxigeno y mayor actividad respiratoria acorde con Handreck y Black (2002), quienes afirman que la porosidad total del sustrato afecta la capacidad de intercambio gaseoso del medio, disminuyendo el contenido de oxígeno que las semillas requieren para germinar.

Ensayos realizados por Maldonado et al. (2002) concluyeron que la capacidad de germinación de las semillas de Lycopersicon chilense estuvieron restringidas a condiciones de abastecimiento hídrico favorable, provocando una reducción en la germinación, debido probablemente a que las enzimas hidrolíticas de los cotiledones no fueron activadas, lo que no desencadenó la activación metabólica de la semilla (Obroucheva y Antipova, 1997; Nonogaki y Morohashi, 1999).

La combinación entre la lámina de riego y los sustratos presentaron diferencias significativas que influyeron en la velocidad y uniformidad de germinación de las semillas, la mezcla TRCQ expresó la mayor respuesta, al igual que la lámina de 1,2. Esto debido a la influencia de la hume- 
Tabla 2. Fórmulas empleadas para el cálculo de las variables de germinación.

\begin{tabular}{|l|l|l|}
\hline \multicolumn{1}{|c|}{ Variable } & \multicolumn{1}{|c|}{ Ecuación } & \multicolumn{1}{c|}{ Unidades } \\
\hline Velocidad media de germinación & $\mathrm{VMG}=\sum\left(\frac{\mathrm{n}_{\mathrm{i}}}{\mathrm{t}_{\mathrm{i}}}\right)$ & Semillas germinadas /día \\
\hline Tiempo medio de germinación & $\mathrm{TMG}=\frac{\mathrm{N}^{*}\left(\mathrm{~A}_{1}+\mathrm{A}_{2}+\mathrm{A}_{\mathrm{x}}\right)}{\left.\mathrm{A}_{1} * \mathrm{~T}_{1}+\mathrm{A}_{2} * \mathrm{~T}_{2}+\mathrm{A}_{\mathrm{x}} * \mathrm{~T}_{\mathrm{x}}\right)}$ & días \\
\hline Porcentaje de germinación & $\mathrm{PG}=\left(\frac{\mathrm{N}}{\mathrm{N}_{\mathrm{s}}}\right) * 100$ & $\%$ \\
\hline
\end{tabular}

$n_{i}=$ Número de semillas germinadas en el i-ésimo día;

$\mathrm{t}_{\mathrm{i}}=$ Tiempo en días, para la germinación en el i-ésimo día.

$\mathrm{N}=$ Número de semillas germinadas.

$\mathrm{N}_{\mathrm{S}}=$ Número de semillas totales.

$A_{1}, A_{2}, \ldots, A_{x}$ : Número de semillas germinadas en el día 1 , en el día 2 , y en el día $x$.

$T_{1}, T_{2}, \ldots, T_{x}$ : Número de días entre la siembra y el primer día 1 de germinación, entre el día 2 y entre el día $x$.

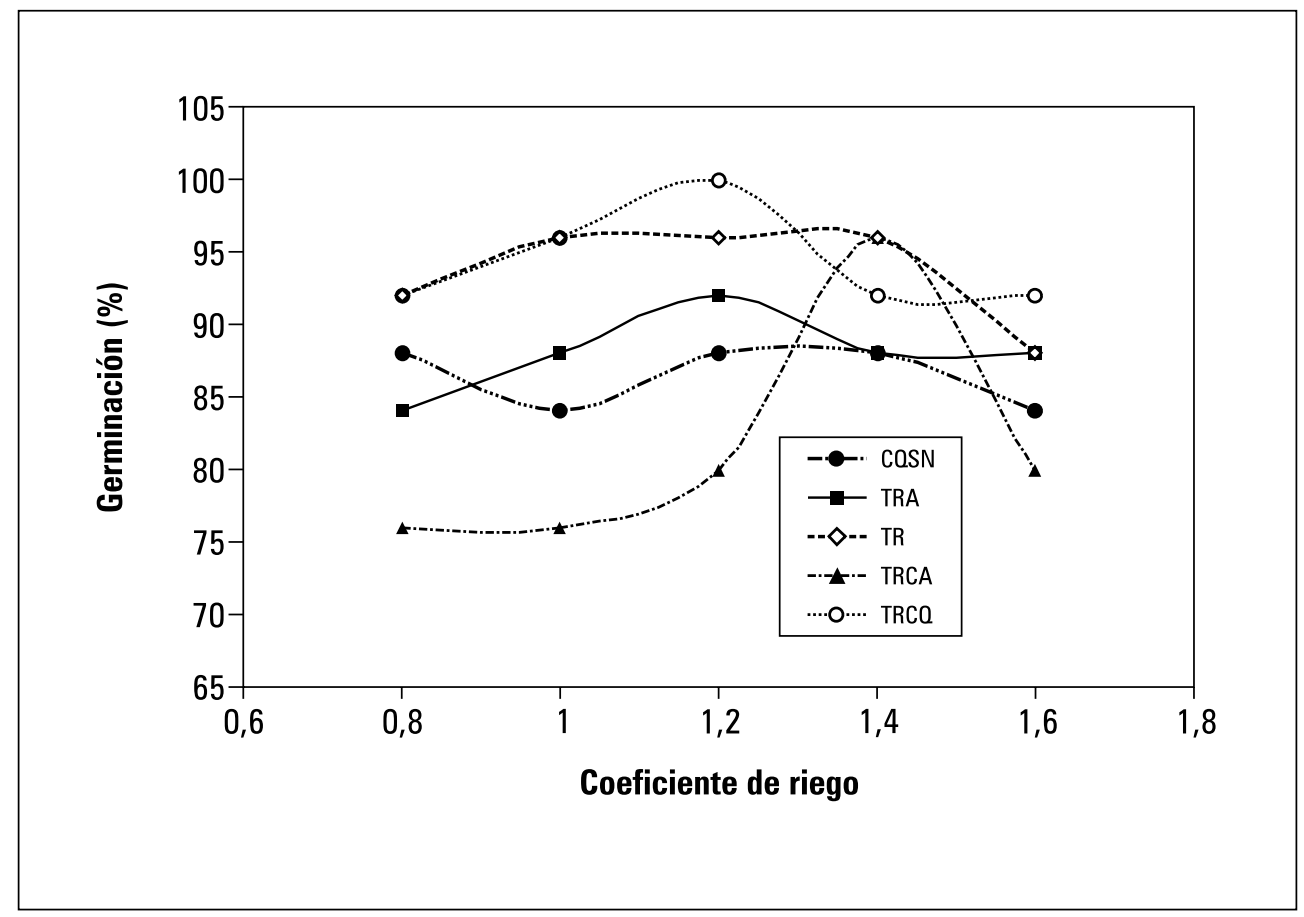

Figura 1: Germinación en plántulas de tomate bajo diferentes láminas de riego sembrados en diferentes sustratos.

dad y la disponibilidad de agua para la semilla en la germinación, que depende de la estructura porosa y la distribución del sustrato.

La velocidad media de germinación (VMG) aumenta en forma directa con la temperatura y la retención de humedad del sustrato, por lo que para este caso se ve que los sustratos con mejor retención presentaron una VMG mayor, debido a que las semillas lograron absorber mayor agua, permitiendo el rompimiento de la cubierta, la emergencia de la radícula y el crecimiento de la plántula. Gul et al. (1995) compararon 10 sustratos, algunos con turba y arena en el cultivo de 
tomate cv. Carpy y obtuvieron plántulas una semana antes que en suelo.

En la variable tiempo medio de germinación, hubo diferencias significativas en el sustrato TRCQ y la lámina de 1,2 indujeron una rápida germinación en comparación a los demás tratamientos, lo que corrobora los resultados de VMG. Se asume que una vez la semilla tiene las condiciones adecuadas de humedad, temperatura y aireación el intercambio de gases entre sustrato y embrión acelera la emergencia.

El retraso de la germinación en los tratamientos con un coeficiente de riego de 1,0 y en los sustratos COSN y TRA se dio por algún tipo de dormancia como mecanismo de defensa de las semillas (Bewley, 1997). Una vez que se rompió esta dormancia la germinación se activó. Según Samperio (2001), la semilla absorbe agua, lo que produce un reblandecimiento en la capa protectora y se inicia el proceso enzimático que activa el crecimiento de la raíz y ésta empieza a alargarse, por tanto, un contenido adecuado de humedad en la semilla garantiza que el proceso germinativo se lleve a cabo en menor tiempo.

Abou Hadid et al. (1988) determinaron el tiempo de germinación de semillas de tomate cv. Alfredo en distintos sustratos, entre ellos uno constituido por arcilla: arena: aserrín (1:1:1) comparadas con semillas cultivadas en camas de suelo semillero, y encontraron que el tiempo de germinación se redujo entre tres a siete días, dependiendo del sustrato usado $y$, además, registraron un menor requerimiento de agua para la germinación de las semillas, con respecto a las camas de suelo semillero.

\section{Altura de las plántulas}

En la altura se encontraron diferencias significativas entre tratamientos. La mayor respuesta en altura se presentó en el sustrato TRCQ y una lámina de 1,2 de la evapotranspiración, con 8,14 $\mathrm{cm}$, frente al sustrato COSN y un coeficiente 0,8 que mostró plántulas de $2,58 \mathrm{~cm}$ (figura 2). La homogeneidad en los sustratos y la VMG influyó en el crecimiento de la plántula.

El desarrollo de las plantas, en su etapa inicial, está directamente relacionado con la uniformidad en la germinación y ésta, a su vez, se puede atribuir exclusivamente a las características propias del sustrato (Fernández-Bravo et al., 2005). Estas condiciones están relacionadas con factores físicos y químicos como el $\mathrm{pH}$, contenido de nutrientes, capacidad de intercambio gaseoso, agua disponible y temperatura, entre otros (Singh, 1998).

Quesada y Méndez (2005) encontraron que la mezcla de diferentes sustratos en la propagación de tomate influye en la altura de la plántula, ya que la deficiente preparación de los materiales se constituye en una limitante física.

\section{Longitud de la raíz principal}

Las semillas de tomate bajo el efecto de las diferentes láminas de riego y sustratos presentaron diferencias significativas en la longitud de raíz, el sustrato TRCQ y el coeficiente de riego de 1,2 generaron la mejor repuesta con una longitud de raíz principal de $15 \mathrm{~cm}$, en relación al sustrato COSN y un coeficiente de 0,8 que mostró 2,46 $\mathrm{cm}$ (figura 3). Esto reitera que el sustrato influye en el crecimiento vegetativo de la plántula y que está directamente relacionado con la retención de humedad, capacidad de aireación y la entrada de agua a la pared celular de la raíz, lo que permite el flujo masivo de solutos y el transporte de nutrientes.

Wilches y Álvarez (2007) encontraron que las plántulas de tomate sometidas a diferentes láminas de riego no presentaron diferencias significativas en la longitud radical; sin embargo, la lámina de coeficiente 2,2 mostró la mayor longitud de raíz principal. El hecho de que no se presentaran diferencias se debe al poco tiempo que tienen las plántulas para expresar el efecto de los tratamientos. 


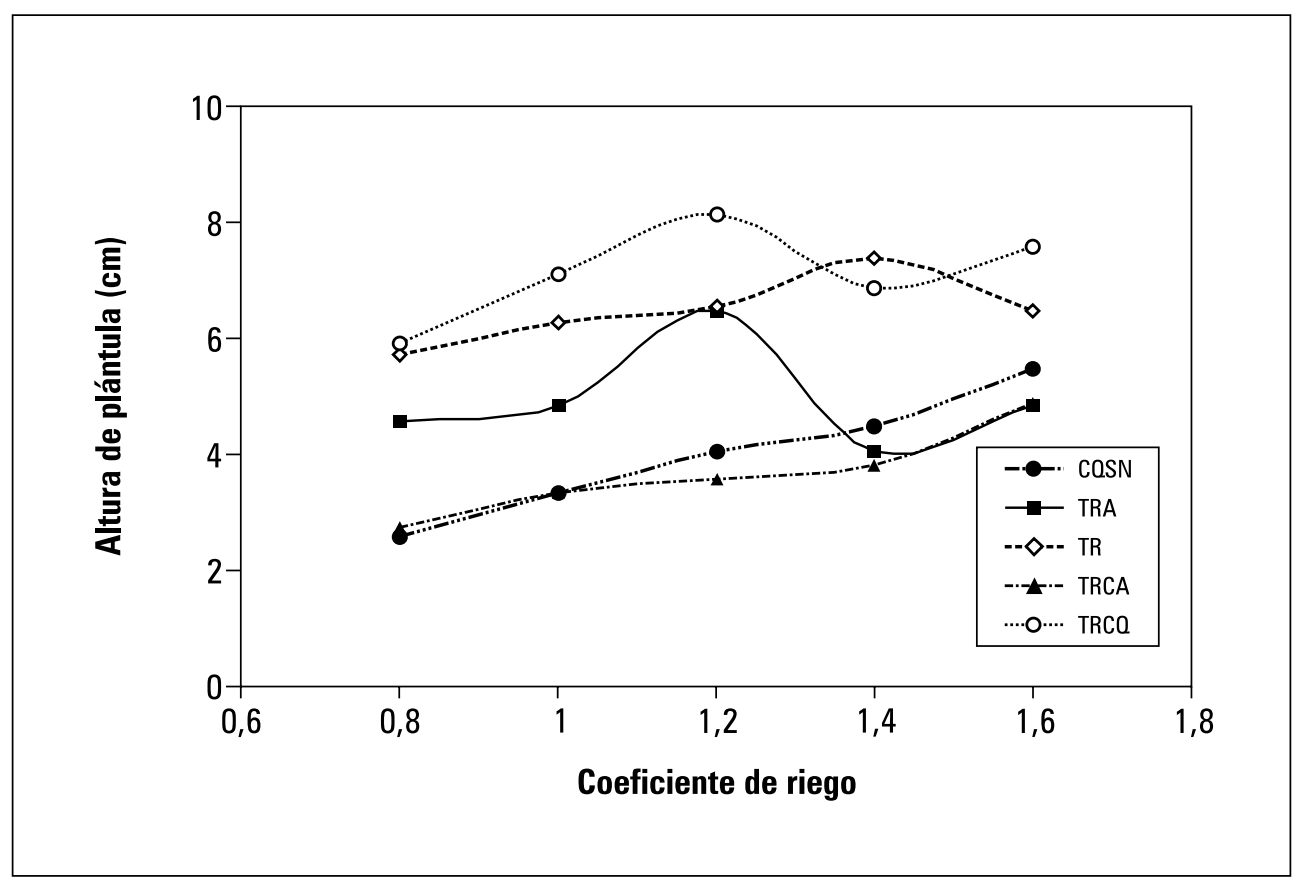

Figura 2. Altura de plántulas de tomate bajo diferentes láminas de riego sembrados en diferentes sustratos.

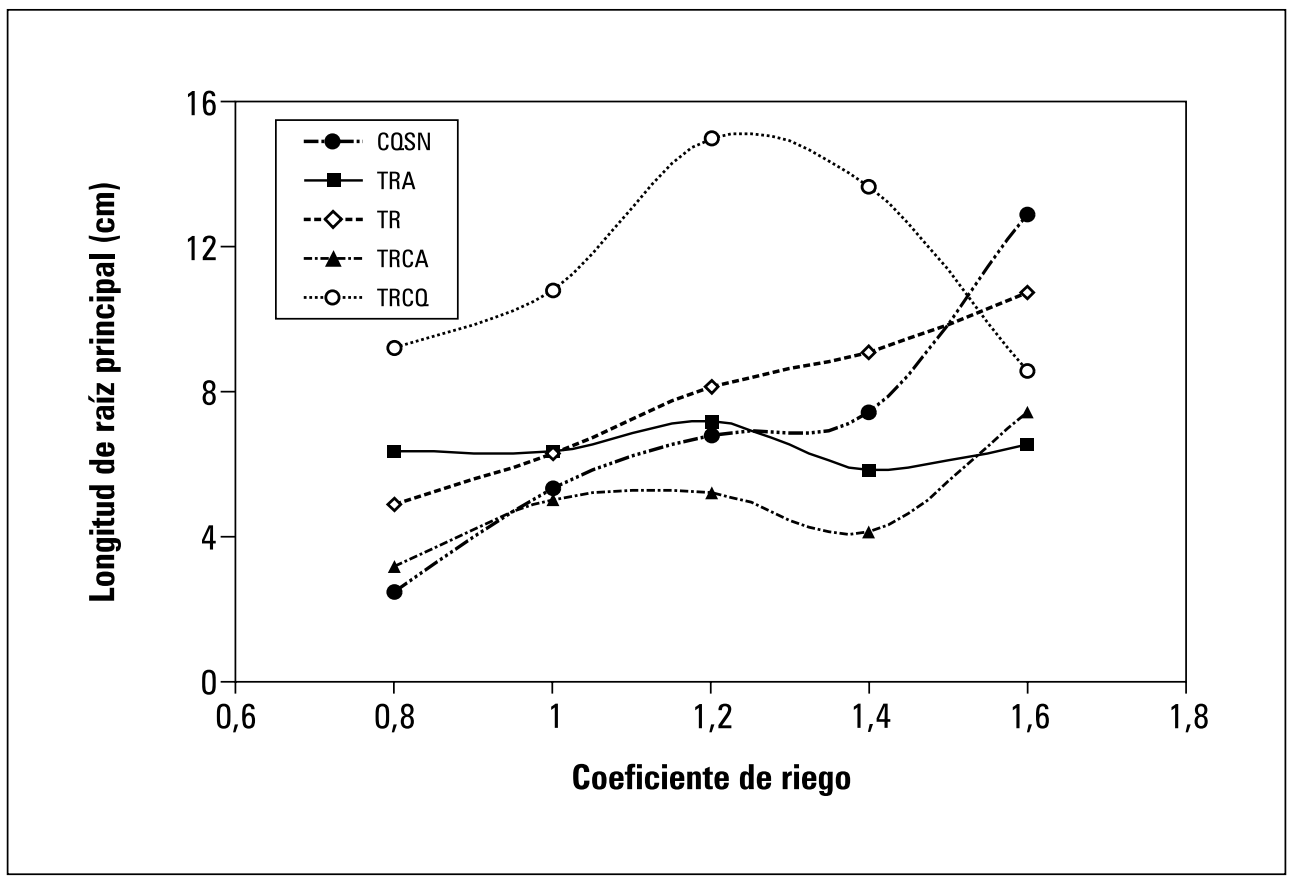

Figura 3. Longitud de raíz de plántulas de tomate bajo diferentes láminas de riego sembradas en diferentes sustratos. 


\section{Área foliar de las plántulas}

Fogg (1967) afirma que la cantidad de fotosíntesis que una planta realiza depende de la superficie de la hoja u órganos fotosintéticos que posea y de la actividad fotosintética por unidad de área de estos tejidos; según Cayón (1992), se obtienen altas producciones por unidad de superficie, con el uso eficiente de la luz durante las etapas iniciales de crecimiento de los cultivos.

Las plántulas de mayor desarrollo mostraron los valores más altos en área foliar, encontrando diferencias significativas entre tratamientos. La mejor respuesta se presentó en el sustrato $\mathrm{TR}$, con un coeficiente de riego 1,6 y el más bajo con 0,8, y el sustrato TRCA, que mostró las plántulas de menor número de hojas.

La plántula en su crecimiento consume mayor cantidad de agua, pero del mismo modo la pier- de rápidamente a través de la evaporación de sus hojas y debe recuperarla, por lo que la turba, al poseer buena retención de humedad, permite un mejor aprovechamiento y flujo de agua a través del xilema hacia la hoja.

Según Barraza (2000), el área foliar depende del número de hojas, de su velocidad de crecimiento $y$ de su tamaño final. Arroyave (2000) afirma que a medida que se desarrolla la planta de tomate las hojas se vuelven más complejas y, por tanto, más funcionales. Según Jarma et al. (1999), las plantas con mayor área foliar y ambiente favorable son capaces de utilizar mejor la energía solar con una fotosíntesis más eficiente.

\section{Peso fresco de los órganos}

El efecto de las láminas de riego y los sustratos en la germinación de tomate influyeron en el crecimiento de hojas, tallo y raíz, encontrando

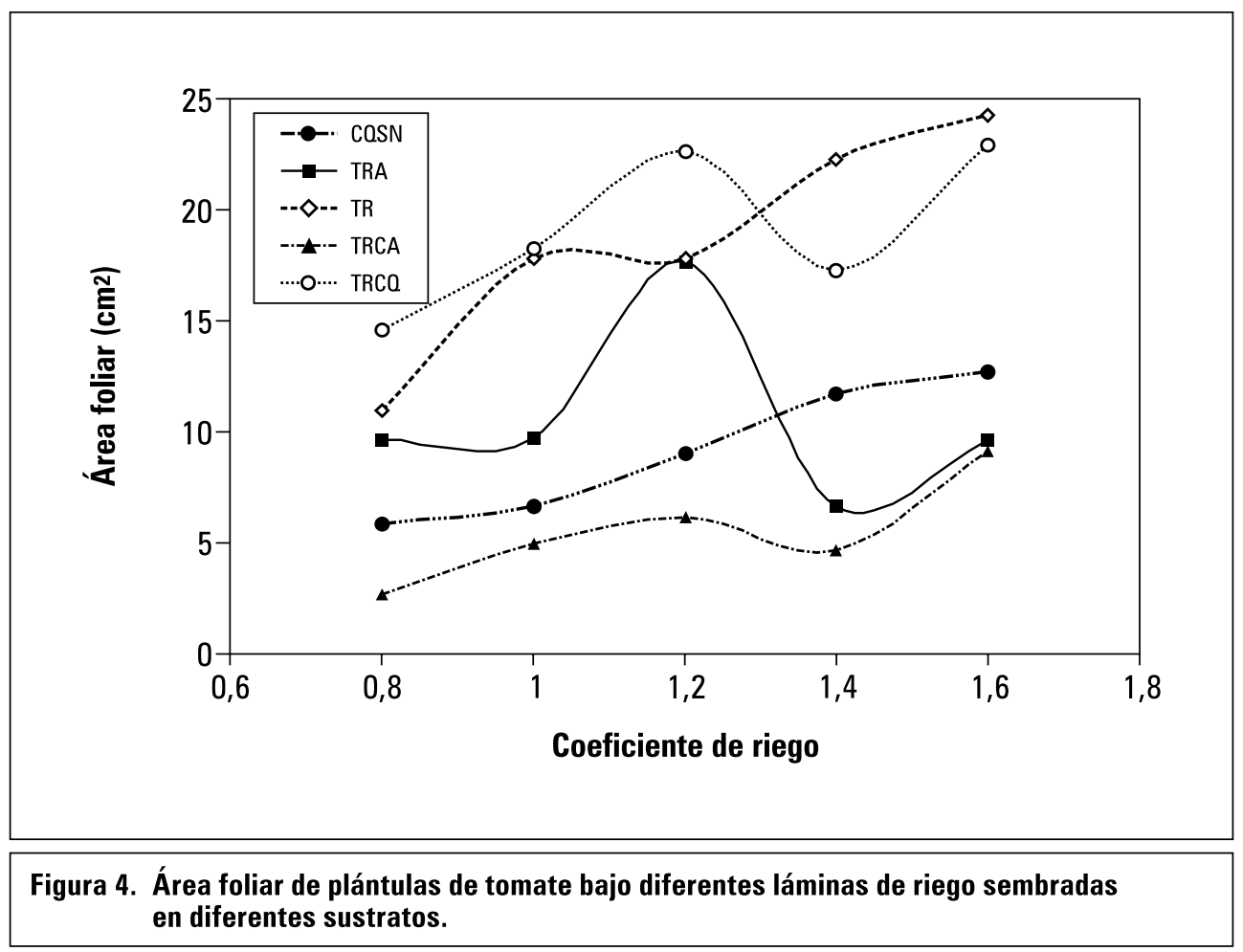


diferencias significativas entre tratamientos. La mayor respuesta en hojas y tallo se obtuvo en el sustrato TRCQ, con un coeficiente de 1,2 frente al sustrato TRCA, con el coeficiente de 0,8 que mostró las plántulas de menor masa (figura 5).

La óptima captación de nutrientes y agua se ve reflejada en los mejores tratamientos, lo que conllevó a una mayor división celular e incremento en masa fresca y a la absorción de oxigeno, que es esencial en el proceso de respiración y de actividad metabólica.

Scagel (1987) menciona que una de las principales funciones del tallo es formar y mantener las hojas y las estructuras de reproducción, conducir agua y nutrientes y almacenar sustancias. A medida que incrementa su tamaño garantiza una mayor acumulación y transporte de solutos en la planta (Alóni, 1987), lo que manifiesta un mayor volumen de agua en sus tejidos, una mayor movilidad de los nutrientes y una actividad fisiológica eficiente.

\section{Peso seco de los órganos}

Con la lámina de 1,2 y el sustrato TRCQ se presentaron diferencias significativas en relación con los demás tratamientos. La lámina de 0,8 y el sustrato TRCA resultó en las plántulas de menor masa seca de hojas y tallo. En el caso de la raíz, el sustrato TRCQ, con un coeficiente de 1,6 , presentó el valor más alto con $0,051 \mathrm{~g}$, frente al sustrato TRCA y un coeficiente de 0,8 que obtuvo plántulas de 0,0013 g (figura 6). Esto se debió al pobre desarrollo alcanzado por la plántula,

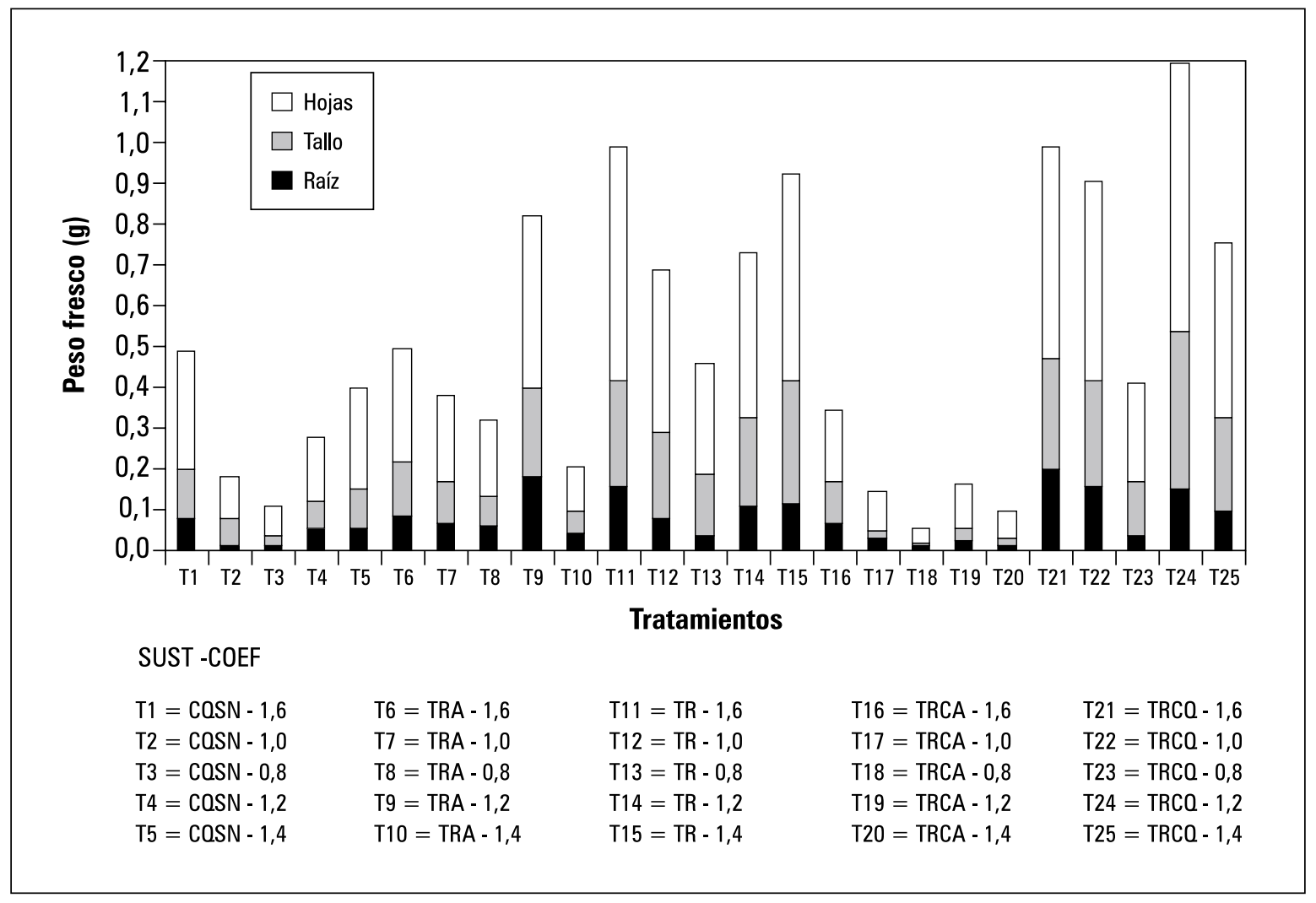

Figura 5. Peso fresco de hojas, tallo y raíz en plántulas de tomate bajo diferentes láminas de riego sembradas en diferentes sustratos. 
debido a la influencia del sustrato, lo que causó una disminución en la actividad metabólica y el almacenamiento de reservas alimenticias y redujo el volumen celular.

Fernández-Bravo et al. (2005) encontraron que plántulas de tomate sembradas en turba obtuvieron los mejores resultados de masa seca de tallo frente a otros sustratos, favoreciendo el crecimiento inicial de la plántula.

Según Tadeo (2000), existe una relación directa entre la masa fresca y la masa seca, en la que una mayor cantidad de agua influye directamente en la masa seca de la planta. De igual forma, una mayor masa seca implica que el agua dentro de los tejidos estará retenida en mayor cantidad y con mayor fuerza.

\section{Análisis económico del sistema de propagación}

Al evaluar los tratamientos económicamente, se encontraron diferencias significativas. El tratamiento más costoso fue el TR con un coeficiente de 1,6 con un valor de $\$ 66,88$ pesos colombianos por plántula, frente al sustrato COSN con un coeficiente de 0,8 , el cual tuvo el menor costo con un valor de $\$ 56,51$. Al comparar los resultados de cada una de las variables con el costo por tratamiento, se ve que la mezcla entre turba y cascarilla quemada es más rentable y económica, pues se obtienen plántulas vigorosas y óptimas para transplante.

El mejor tratamiento costó $\$ 63,69$ y está constituido por la combinación de la lámina de riego 1,2 y el sustrato TRCO. De igual forma, fue el de

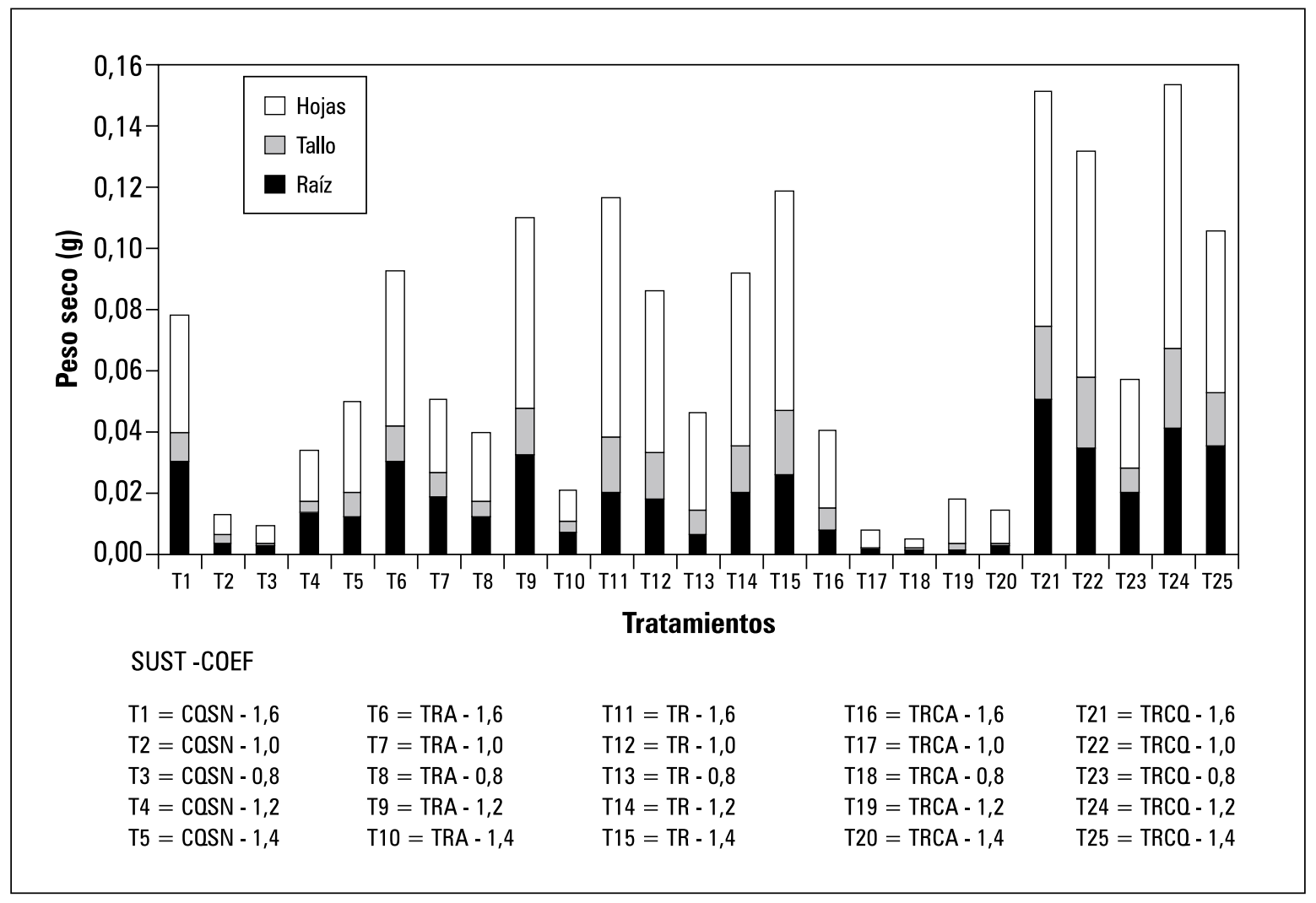

Figura 6. Peso seco de hojas, tallo y raíz en plántulas de tomate bajo diferentes láminas de riego sembradas en diferentes sustratos. 
mejor eficiencia económica, lo que significa que generó mayor masa seca por unidad de masa de agua empleada.

\section{CONCLUSIONES}

- La mezcla de turba rubia con cascarilla quemada y la lámina de 1,2 presentó los mejores resultados en altura, longitud de raíz, peso fresco y seco de hojas y tallo.

- La lámina de 0,8 y el sustrato turba rubia obtuvo la mejor eficiencia en masa seca por agua consumida.

- El tratamiento óptimo económicamente fue el de la lámina de riego 1,2 y la mezcla de turba rubia con cascarilla quemada.

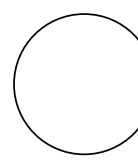

REFERENCIAS BIBLIOGRÁFICAS

Abou Hadid, A.; S. El Beltagy y H. El Saied. 1988. Soil mixture for tomato transplants. Egypt. J. Hort. 15, 23-28.

Alóni, B. y E. Pressman. 1987. The effects of salinity and gibberelic acid on blackheart disorder in celery. Agron. J. 79, 205-208.

Arroyave, H. 2000. Siembre los híbridos Petoseed. La compañía de las semillas híbridas. Bogotá. pp. 120.

Barraza, F.V. 2000. Crecimiento del Chile Manzano (Capsicum pubescens R. y P.) en cuatro soluciones nutritivas bajo invernadero. Tesis de Maestría, Ciencias en Horticultura, Universidad Autónoma de Chapingo, México.

Benech, A.; R.L. Fenne y P.J. Edwards. 1992. Changes in dormancy level in Sorghum halepense seeds induced by water stress during seed development. Funct. Ecol. 6, 596-605.

Bewley, J.D. 1997. Seed germination and dormancy. Plant Cell 9, 1055-1066.

Castilla, N. 1995. Manejo del cultivo intensivo del tomate. pp. 189-226. En: Nuez, F. (ed.). El cultivo del tomate. Ediciones Mundi-Prensa, Madrid.

Cayón, G. 1992. Fotosíntesis y productividad de cultivos. Rev. Comalfi 19(2), 23-31.

Dubreucq, B.; N. Berger; E. Vincent; M. Boisson; M. Caboche y L. Lepiniec. 2000. The Arabidopsis AtEPR1 extensin-like gene is specifically expressed in endosperm during seed germination. Plant J. 23, 643-652.
Ellis, R. y T.D. Hong. 1994. Desiccation tolerance and potential longevity of developing seeds of rice (Oryza sativa L.). Ann. Bot. 73, 501-506.

FAO. 2007. FAOStat estadística databases, agricultura, En: http://faostat.fao.org/site/340/default.aspx; consulta: 4 de febrero de 2008 .

Fernández-Bravo, C.; N. Urdaneta; W. Silva; H. Poliszu y M. Marín. 2005. Germinación de semillas de tomate (Lycopersicon esculentum Mill.) cv. Río Grande sembradas en bandejas plásticas, utilizando distintos sustratos. Rev. Fac. Agron. LUZ 23(2), 188-195.

Fogg, G.E. 1967. El crecimiento de las plantas. Editorial Universitaria de Buenos Aires, Argentina.

Foley, M.E. y S.A. Fennimore. 1998. Genetic basis for seed dormancy. Seed Sci. Res. 8, 173-182.

Gómez, O.; A. Casanova; H. Laterot e Y.G. Anais. 2000. Mejora genética y manejo del cultivo del tomate para la producción en el Caribe. Instituto de Investigaciones Hortícolas "Liliana Dimitrova", La Habana, Cuba.

Gul, A.; A. Sevgican; F. Tognoni; G. Serra y A. Monteiro. 1995. Effect of growing media on glasshouse tomato yield and quality. Acta Hort. 303, 145-150

Gutterman, Y. 2000. Maternal effects on seed during development. pp. 59-84. En: Fenner, M. (ed.). Seed: the ecology of regeneration in plant communities. CAB International, Wallingford, UK.

Handreck, K. y N. Black. 2002. Growing media for ornamental plants and turf. $3^{\text {th }}$ ed. UNSW Press, Australia. 
Hay, F.R. y R.J. Probert. 1995. Seed maturity and the effects of different drying conditions on desiccation tolerance and seed longevity in foxglove (Digitalis purpurea L.). Ann. Bot. 76, 639-647.

Hong, T.D. y R.H. Ellis. 1992. Development of desiccation tolerance in Norway maple (Acer platanoides L.) seeds during maturation drying. Seed Sci. Res. 2, 169-172.

Jarma, A.; C. Buitrago y S. Gutiérrez. 1999. Respuesta del crecimiento de la habichuela (Phaseolus vulgaris L. var. BlueLake) a tres niveles de radiación incidente. Rev. Comalfi 26(1-3), 62-73.

Maldonado, C.; E. Pujado y F.A. Squeo. 2002. El efecto de la disponibilidad de agua durante el crecimiento de Lycopersicon chilense sobre la capacidad de sus semillas para germinar a distintas temperaturas y concentraciones de manitol y $\mathrm{NaCl}$. Rev. Chil. Histor. Natur. 75, 651-660.

Nonogaki, H. y Y. Morohashi. 1999. Temporal and spatial pattern of the development of endo-ß-mannanase activity in germinating and germinated lettuce seeds. J. Exp. Bot. 50, 1307-1313.

Obroucheva, N.V. y O.V. Antipova. 1997. Physiology of the initiation of seed germination. Russ. J. Plant Physiol. 44, 250-264.

Pallas, J.E.; J.R. Stansell y R.R. Bruce. 1977. Peanut seed germination as related to soil water regime during pod development. Agron. J. 69, 381-383.

Quesada, G. y C. Méndez. 2005. Análisis fisicoquímico de materias primas y sustratos de uso potencial en almácigos de hortalizas. Rev. Agr. Trop. 35, 1-13.
Riggle, D. 1998. Vermicomposting research and education. BioCycle 39, 54-56.

Samperio, G. 2001. Germinación de semillas: Manual de divulgación para uso en instituciones de educación. Toluca, Estado de México. En: http://www.rlc.fao. org/es/agricultura/aup/pdf/mexico.pdf; consulta: febrero de 2008.

Scagel, R.F. 1987 El reino vegetal. 2a ed. Ediciones Omega, Barcelona.

Singh, B.P. y U.M. Sainju. 1998. Soil physical and morphological properties and root growth. HortScience 33(6), 966-971.

Subler, S.; C.A. Edwards y J.D. Metzger. 1998. Comparing vermicomposts and composts. BioCycle 39, 63-66.

Tadeo, F. 2000. Fisiología de las plantas y el estrés. pp. 481-498. En: Azcon-Bieto, J. y M. Talón (eds.). Fundamentos de fisiología vegetal. Mc Graw-Hill/ Interamericana de España, Barcelona.

Wechsberg, G.E.; C.M. Bray y R.J. Probert. 1994. Expression of dehydrin-like proteins in orthodox seeds of Ranunculus scleratus during development and water stress. Seed Sci. Res. 4, 241-246.

Wilches, F. y J. Álvarez. 2007. Tamaños de alveolo y diferentes láminas de riego en obtención de plántulas de tomate (Lycopersicon esculentum Mill.). Trabajo de grado. Facultad de Ciencias Agropecuarias, UPTC, Tunja. 